\title{
Steiner triple systems and existentially closed graphs
}

\author{
A.D. Forbes, M.J. Grannell and T.S. Griggs \\ Department of Pure Mathematics \\ The Open University \\ Walton Hall \\ Milton Keynes MK7 6AA \\ UNITED KINGDOM \\ tonyforbes@ltkz.demon.co.uk \\ m.j.grannell@open.ac.uk \\ t.s.griggs@open.ac.uk
}

Submitted Dec 20, 2004; Accepted: Apr 7, 2005; Published: Aug 30, 2005

Mathematics Subject Classifications: 05C99, 05B07

\begin{abstract}
We investigate the conditions under which a Steiner triple system can have a 2- or 3-existentially closed block intersection graph.
\end{abstract}

\section{Introduction}

A graph $G=(V, E)$, where $V$ is the set of vertices and $E$ is the set of edges, is said to be $n$-existentially closed, or $n$-e.c., if for every $n$-element subset $S$ of $V$, and for every subset $T$ of $S$, there exists a vertex $x \notin S$ which is adjacent to every vertex in $T$, and is not adjacent to any vertex in $S \backslash T$. These graphs were first studied by Caccetta, Erdős and Vijayan, [6], although Erdôs and Rényi, [8], had previously proved the interesting result that for any fixed value of $n$, almost all graphs are $n$-e.c. But relatively few specific examples of $n$-e.c. graphs are known for $n \geq 2$.

A strongly regular graph $\operatorname{SRG}(v, k, \lambda, \mu)$ is a regular graph of degree $k$ on $v$ vertices with the property that every pair of adjacent vertices has $\lambda$ common neighbours and every pair of non-adjacent vertices have $\mu$ common neighbours. An important class of strongly regular graphs are the Paley graphs. The Paley graph of order $q$, where $q \equiv$ $1(\bmod 4)$ is a prime power, is the graph with vertex set $\operatorname{GF}(q)$, the Galois field of order $q$, and the edge set is the set of pairs $\{x, y\}$ where $x-y$ is a square. It is an $\operatorname{SRG}(q,(q-1) / 2,(q-5) / 4),(q-1) / 4)$. Ananchuen and Caccetta, [1], proved that all Paley graphs with at least 29 vertices are 3-e.c.; see also [3] and [5]. In [2], Baker, Bonato and Brown constructed 3-e.c. strongly regular graphs $\operatorname{SRG}\left(q^{2},\left(q^{2}-1\right) / 2,\left(q^{2}-5\right) / 4,\left(q^{2}-1\right) / 4\right)$ 
using affine planes of order $q \geq 7$. In [4], Bonato, Holzmann and Kharagani studied 3-e.c. strongly regular graphs obtained from Hadamard matrices.

Another area of Design Theory where strongly regular graphs appear is as the block intersection graphs of Steiner triple systems. In this paper we study the adjacency properties of these graphs. These naturally relate to questions concerning configurations. Using this approach we are able to prove that the block intersection graph of every Steiner triple system, with the exception of the unique systems on 7 and 9 points, is 2-e.c. Furthermore, we obtain a characterization of those Steiner triple systems whose block intersection graphs are 3-e.c. This characterization implies that such graphs can exist only for a limited range of orders of Steiner triple system, possibly even just one order; 19 points. However, these graphs do exist and we are able to identify two 3-e.c. strongly regular graphs with 57 vertices, an order for which such a graph was previously unknown. The fact that there are a finite number of these graphs raises the question of whether it is possible to enumerate them. We discuss this in the conclusion but we think that it is likely to be quite difficult. The evidence suggests that 3-e.c. strongly regular graphs which occur as the block intersection graphs of Steiner triple systems are a rare and elusive species and likely to remain so. But it is a tantalizing problem in Design Theory. In the next section we review basic facts about Steiner triple systems and configurations.

\section{$2 \quad$ Steiner triple systems}

A Steiner triple system of order $v, \operatorname{STS}(v)$, is a pair $(V, \mathcal{B})$ where $V$ is a set of cardinality $v$ of elements, or points, and $\mathcal{B}$ is a collection of triples, also called blocks, which has the property that every pair of distinct elements of $V$ occurs in precisely one triple. It is well known that an $\operatorname{STS}(v)$ exists if and only if $v \equiv 1$ or $3(\bmod 6)$. Such values are called admissible. An independent set in a Steiner triple system $(V, \mathcal{B})$ is a subset of $V$ no three elements of which form a block. Clearly, any subset of an independent set is also an independent set and hence, for any given Steiner triple system $D$, interest focuses on the size of an independent set of maximum cardinality in $D$. We denote this cardinality by $I(D)$. We put $I(v)=\max \{I(D): D$ is an $\operatorname{STS}(v)\}$. The block intersection graph of a Steiner triple system is the graph whose vertices are the set of blocks of the STS $(v)$ with two vertices being joined by an edge if the blocks they represent have a non-empty intersection. It is an $\operatorname{SRG}(v(v-1) / 6,3(v-3) / 2,(v+3) / 2,9)$.

In the context of a Steiner triple system, a configuration is a collection of triples which has the property that every pair of distinct elements occurs in at most one triple. If $\mathcal{C}$ is a configuration, we denote the set of points by $P(\mathcal{C})$, the number of points by $p(\mathcal{C})$ and the number of blocks by $b(\mathcal{C})$. Two configurations $\mathcal{C}$ and $\mathcal{D}$ are said to be isomorphic, in symbols $\mathcal{C} \cong \mathcal{D}$, if $p(\mathcal{C})=p(\mathcal{D}), b(\mathcal{C})=b(\mathcal{D})$ and there exists a one-to-one mapping $\phi: P(\mathcal{C}) \rightarrow P(\mathcal{D})$ such that for each triple $T \in \mathcal{C}, \phi(T)$ is a triple in $\mathcal{D}$. Two STS $(v) \mathrm{s}$, $(V, \mathcal{B})$ and $\left(V^{\prime}, \mathcal{B}^{\prime}\right)$ are isomorphic if $\mathcal{B} \cong \mathcal{B}^{\prime}$. The degree of an element, either in a configuration or a Steiner triple system, is the number of blocks which contain it.

General information on configurations and Steiner triple systems can be found in the survey paper, [13]. We give here the definitions and results that are relevant for 
this investigation. The number of non-isomorphic $n$-block configurations for $1 \leq n \leq$ 10 is known, [10]. Specifically, there are two 2-block configurations and five 3-block configurations. Below, we list these as well as some further specific configurations that we refer to in the rest of the paper. We give them their standard names and their canonical labellings in the point set $\{0,1, \ldots\}$. A pair of triples which do not intersect are said to be parallel. A set of $n \geq 2$ triples every pair of which are parallel is said to be an $n$-parallel class or $n$-p.c. As a matter of notational convenience, set brackets and commas are usually omitted from the description of the pairs and triples; thus, for example, the block $\{x, y, z\}$ is denoted simply by $x y z$.

\begin{tabular}{|l|l|l|}
\hline Symbol & Name & Blocks \\
\hline$A_{1}$ & 2-p.c. & $\{012,345\}$ \\
$A_{2}$ & & $\{012,034\}$ \\
$B_{1}$ & 3-p.c. & $\{012,345,678\}$ \\
$B_{2}$ & hut & $\{012,034,567\}$ \\
$B_{3}$ & 3-star & $\{012,034,056\}$ \\
$B_{4}$ & 3-path & $\{012,034,156\}$ \\
$B_{5}$ & triangle & $\{012,034,135\}$ \\
$C_{14}$ & & $\{012,034,135,246\}$ \\
$C_{16}$ & Pasch & $\{012,034,135,245\}$ \\
$D_{1}$ & mitre & $\{012,034,135,236,456\}$ \\
\hline
\end{tabular}

Configurations may be either constant or variable. A constant configuration is one which, for each admissible value of $v$, occurs the same number of times in every $\operatorname{STS}(v)$. Thus, the formula for the number of occurrences of a constant configuration is a function of $v$ only. Both 2-block and all five 3-block configurations are constant. A variable configuration means that for some values of $v$ there are at least two (necessarily nonisomorphic) STS $(v)$ s containing different numbers of the configuration. Of the 16 4-block configurations, five are constant and eleven are variable. The formulae for the number of occurrences of all of the variable configurations can be expressed in terms of the order $v$ and the number of occurrences of any one of them. In practice it is natural to choose the Pasch configuration if only because it is the 'tightest', being the unique 4-block configuration containing the least number (six) of points.

The 4-block Pasch configuration and the 5-block mitre configuration play key roles in the theory of configurations in Steiner triple systems. The construction of systems which avoid either or both of them is of interest. The spectrum of values for which there exists a so called anti-Pasch $\operatorname{STS}(v)$ is now known to be the set of all admissible $v$ except $v=7$ and 13, [16] and [15]. Anti-mitre $\operatorname{STS}(v)$ s exist for all admissible $v$ except $v=9,[7]$, [11], [12] and [22]. Systems which are both anti-Pasch and anti-mitre are called 5-sparse. No 5sparse $\operatorname{STS}(v)$ exist for $v=7,9,13$ or 15 but there does exist both a 5 -sparse STS(19) and a 5-sparse $\operatorname{STS}(33)$. The cases $v=21,25,27$ and 31 are undecided. Recently, Fujiwara, [12], has proved that 5 -sparse $\operatorname{STS}(v)$ exist for all $v \equiv 1$ or $19(\bmod 54)$ with the possible exception of $v=109$. 


\section{Systems with 2-e.c. block intersection graphs}

Theorem 3.1 The block intersection graph of a Steiner triple system of order $v$ is 2existentially closed if and only if $v \geq 13$.

Proof. Consider an $\operatorname{STS}(v)$ with $v \geq 13$. There are two 2-block configurations that occur in the $\operatorname{STS}(v)$; namely (i) a pair of intersecting blocks, $\{a b c, a d e\}$, or (ii) a pair of parallel blocks, $\{a b c, d e f\}$. For each of these configurations we need to show that there exists (a) a block that intersects both blocks of the configuration, (b) a block that intersects exactly one block of the configuration, and (c) a block that is parallel to both blocks of the configuration. We consider each case in turn.

Case (i)(a). There are at least three blocks that contain the point $a$. Hence there exists a suitable block, afg, say, that intersects both $a b c$ and ade.

Case (i)(b). There are at least four blocks that contain the point $b$, and therefore at least one of them must avoid the points $a, d$ and $e$. Hence there exists a block that intersects $a b c$ but not ade.

Case (i)(c). Choose a point, $h \notin\{a, b, c, d, e\}$. There are at least six blocks that contain $h$, and therefore at least one of them must avoid $a b c$ and ade.

Case (ii)(a). A block that intersects both $a b c$ and def is the block that contains the pair ad.

Case (ii)(b). There are at least five blocks that contain the point $a$, and therefore at least one of them must avoid block def.

Case (ii)(c). Choose a point, $i \notin\{a, b, c, d, e, f\}$. If $v \geq 15$, there are at least seven blocks containing $i$, and hence there must be a block that avoids both $a b c$ and def. If $v=13$, we argue as follows. Partition the points of the STS(13) into two sets, $A$ and $B$, where $A=\{a, b, c, d, e, f\}$. Let $\nu(\alpha, \beta)$ denote the number of blocks that contain precisely $\alpha$ points of $A$ and $\beta$ points of $B$. Clearly $\nu(3,0)=2$ and $\nu(2,1)=9$. Hence to account for the $42 A B$ pairs we must have $\nu(1,2)=(6 \cdot 7-2 \cdot 9) / 2=12$. There are 26 blocks in an STS(13). Therefore $\nu(0,3)=26-2-9-12=3$ and each of these three blocks will be disjoint from both $a b c$ and def.

The above proves the 'if' part of the theorem. To prove the 'only if' part note first that the block intersection graph of the unique $\operatorname{STS}(7)$ is the complete graph, $K_{7}$. In the unique STS(9), case (ii)(c) fails. Any block which intersects abc also intersects def.

\section{Systems with 3-e.c. block intersection graphs}

We now turn our attention to the existence of Steiner triple systems having 3-existentially closed block intersection graphs. First we state and prove two lemmas which will simplify the proof of the main Theorem 4.1.

Lemma 4.1 Let $v \geq 19$ and suppose $\mathcal{X}$ is a three-block configuration in a Steiner triple system of order $v$. Let $x$ be a point in $\mathcal{X}$. Then there exists a block that intersects each of the blocks of $\mathcal{X}$ that contain $x$ and none of the blocks of $\mathcal{X}$ that do not contain $x$. 
Proof. Since $v \geq 19$, there are at least nine blocks containing $x$. Also $\mathcal{X}$ has at most six points not in any of the blocks of $\mathcal{X}$ that contain $x$. Hence there exists at least one block which intersects all the blocks of $\mathcal{X}$ that contain $x$ and is disjoint from all the blocks of $\mathcal{X}$ that do not contain $x$.

Lemma 4.2 Let $v \geq 19$ and suppose $\mathcal{X}$ is a three-block configuration in a Steiner triple system of order $v$. Suppose also that $\mathcal{X}$ has at most eight points. Then there exists a block that is disjoint from $\mathcal{X}$.

Proof. Let $y$ be a point in the $\operatorname{STS}(v)$ which is not in $\mathcal{X}$. Then, since $v \geq 19$, there exist at least nine blocks containing $y$ and, since $\mathcal{X}$ contains at most eight points, there is at least one block (containing $y$ ) that is disjoint from $\mathcal{X}$.

Theorem 4.1 The block intersection graph of a Steiner triple system of order v, STS(v), is 3-existentially closed if and only if all of the following criteria are satisfied:

(i) the STS $(v)$ contains no subsystem STS(7),

(ii) the $S T S(v)$ contains no subsystem STS(9),

(iii) for every set of three parallel blocks, there exists a block which intersects all three.

Proof. Since an $n$-existentially closed graph is $(n-1)$-existentially closed, the theorem is trivially true for $v=7$ and 9. There are two non-isomorphic STS(13)s and 80 nonisomorphic STS(15)s, [17], and a straightforward computation establishes that none of these have 3-existentially closed block intersection graphs. Both of the STS(13)s and 76 of the 80 STS(15)s violate criterion (iii) and the four other STS(15)s, \#1, \#2, \#3 and \#16 in the standard listing in [17] contain a subsystem STS(7).

So now assume that $v \geq 19$. First we deal with the 'if' part of the theorem. The proof is similar to that of Theorem 3.1 but with a somewhat larger number of cases to check.

There are five 3-block configurations that occur in the STS $(v)$; namely (i) three intersecting blocks, a 3-star, $\{a b c$, ade, afg $\}$; (ii) a triangle, $\{a b c$, ade, bdf $\}$; (iii) a 3-path, $\{a b c, a d e, b f g\}$; (iv) a 2-path and a disjoint block (also known as a 'hut'), $\{a b c, a d e, f g h\}$; (v) a 3-p.c., $\{a b c, d e f, g h i\}$. For each of these five configurations we need to show that there exists (a) a block that intersects all three blocks of the configuration; (b) blocks that intersect two given blocks of the configuration but not the third; (c) blocks that intersect a given block of the configuration but not the other two; and (d) a block that is parallel to all three blocks of the configuration. We consider each case separately.

Lemma 4.1 immediately deals with cases (i)(a), (i)(c), (ii)(b), (ii)(c), (iii)(c), (iv)(c) and $(\mathrm{v})(\mathrm{c})$, and Lemma 4.2 with (i)(d), (ii)(d), (iii)(d) and (iv)(d).

Case (i)(b). Without loss of generality, consider the two blocks $a b c$ and ade. The set of pairs $\{b d, b e, c d, c e\}$ yields a set, $\mathcal{F}$, of four blocks. If all four blocks of $\mathcal{F}$ intersect $a f g$, the third block of the $B_{3}$, then $\{a b c$, ade, $a f g\} \cup \mathcal{F}$ is an $\operatorname{STS}(7)$. Since this possibility is ruled out by criterion (i), there exists a block in $\mathcal{F}$ that intersects $a b c$ and ade but avoids afg.

Case (ii)(a). The block containing the pair af intersects all three blocks of the triangle. Case (iii)(a). The block containing the pair af intersects all three blocks of the 3-path. 
Case (iii)(b). For pairs of blocks $\{a b c, a d e\}$ and $\{a b c, b f g\}$, Lemma 4.1 ensures that there exist blocks $A$ and $B$ such that $a \in A, b \in B$ and $A \cap b f g=B \cap$ ade $=\emptyset$. The only other case is the pair of blocks $\{a d e, b f g\}$. Here we note that the blocks generated by the pairs $d f$ and $d g$ cannot both intersect block $a b c$.

Case (iv)(a). The block containing the pair af intersects all three blocks of the hut configuration.

Case (iv)(b). For the pair of blocks $\{a b c, a d e\}$, Lemma 4.1 ensures that there exists a block which contains $a$ and avoids $f g h$. For the pair $\{a b c, f g h\}$ we note that at least one of the blocks generated by the pairs $\{b f, b g, b h\}$ must be disjoint from block ade. The pair $\{a d e, f g h\}$ is handled similarly.

Case (v)(a). This is criterion (iii) in the statement of the theorem.

Case (v)(b). Without loss of generality we may consider just the pair of blocks $a b c$ and $d e f$. The set of pairs $\{a d, a e, a f, b d, b e, b f, c d, c e, c f\}$ yields a set, $\mathcal{G}$ of nine blocks. If all of these blocks intersect with $g h i$, the third block of the 3-p.c., then $\{a b c, d e f, g h i\} \cup \mathcal{G}$ is an $\operatorname{STS}(9)$, contrary to criterion (ii). Hence at least one block of $\mathcal{G}$ is disjoint from $g h i$.

Case (v)(d). If $v \geq 21$, an argument similar to that in the proof of Lemma 4.2 shows that there exists a block which is disjoint from the 3-p.c. So let $v=19$ and partition the points of the STS(19) into two sets, $A$ and $B$, where $A=\{a, b, c, d, e, f, g, h, i\}$. Let $\nu(\alpha, \beta)$ denote the number of blocks which contain $\alpha$ points of $A$ and $\beta$ points of $B$. Let $\nu(3,0)=n$. Then by a simple computation we have $\nu(2,1)=36-3 n, \nu(1,2)=9+3 n$ and $\nu(0,3)=12-n$. Hence $n \leq 12$. However, $n=12 \mathrm{implies}$ that $A$ is the point set of an $\operatorname{STS}(9)$, which is ruled out by criterion (ii). Therefore $n \leq 11$ and $\nu(0,3) \geq 1$, as required.

That completes the 'if' part of the proof. For the reverse implication, suppose that a Steiner triple system $S$ has a 3 -existentially closed block intersection graph. Then observe that criterion (iii) follows trivially, and the arguments given in cases (i)(b) and (v)(b), above, are reversible, implying that $S$ cannot have a sub-STS(7) nor a sub-STS(9).

We now determine an upper limit of $v$ for which an $\operatorname{STS}(v)$ can have a 3-existentially closed block intersection graph.

Theorem 4.2 If $v \geq 33$, a Steiner triple system of order $v$ cannot have a 3-existentially closed block intersection graph.

Proof. The maximum cardinality of an independent set in a Steiner triple system of order $v$ is

$$
I(v)= \begin{cases}\frac{v+1}{2} & \text { if } v \equiv 3 \text { or } 7(\bmod 12), \\ \frac{v-1}{2} & \text { if } v \equiv 1 \text { or } 9(\bmod 12),[19] .\end{cases}
$$

Let $a b c$, def be a pair of parallel blocks in an $\operatorname{STS}(v)$. Such a pair always exists if $v \geq 9$. The set of pairs $\{a d, a e, a f, b d, b e, b f, c d, c e, c f\}$ yields a set, $\mathcal{F}$, of nine blocks. Now consider the 11-block configuration, $\mathcal{C}$, consisting of $\mathcal{F}$ together with the two original blocks 
$a b c$, def. Then $p(\mathcal{C}) \leq 15$. Hence if $v-15>I(v)$, there exists a block $g h i$ which is parallel to both $a b c$, def, and such that criterion (iii) of Theorem 4.1 is violated. Performing the calculation shows that $v \geq 33$.

The above theorem limits the range of orders for which an $\operatorname{STS}(v)$ can have a 3-e.c. block intersection graph to $v=19,21,25,27$ and 31 . The next theorem eliminates the last two values from this list.

Theorem 4.3 A Steiner triple system of order 27 or 31 cannot have a 3-existentially closed block intersection graph.

Proof. Consider the two orders separately.

(a) $v=31$. The maximum cardinality of an independent set is 16 .

The number of $C_{14}$ configurations in an $\operatorname{STS}(v)$ is given by

$$
c_{14}=\frac{1}{4} v(v-1)(v-3)-6 p
$$

where $p$ is the number of Pasch configurations, [14]. So $c_{14}=0$ if and only if $p=$ $v(v-1)(v-3) / 24$, and this can only occur if $v=2^{n+1}-1$ and the $\operatorname{STS}(v)$ is the point-line design of the projective geometry $\mathrm{PG}(n, 2)$; in this case $\mathrm{PG}(4,2)$; see [21]. But since this contains $\operatorname{STS}(7)$ subsystems, criterion (i) of Theorem 4.1 is violated.

Otherwise $c_{14}>0$ and the argument proceeds as in the proof of the previous theorem. Let $\{a b c, d e f, a d j, c e j\}$ be a $C_{14}$ configuration and define the configuration $\mathcal{C}$ precisely as in the proof of Theorem 4.2. Then in this case, since both adj and cej are blocks, $p(\mathcal{C}) \leq 14$. The set of the remaining 17 or more points is not an independent set and therefore contains a block $g h i$ which is parallel to both $a b c$, def, and such that criterion (iii) of Theorem 4.1 is violated.

(b) $v=27$. The maximum cardinality of an independent set is 14 . We consider various subcases for the size of a maximum independent set, $I(D)$.

(i) $I(D)=14$. Partition the points of the $\mathrm{STS}(27)$ into two sets $A$ and $B$ where $B$ is a maximum independent set. Then, using the same notation as in the proofs of Theorems 3.1 and $4.1, \nu(1,2)=91$ and $\nu(3,0)=26$. It follows that the collection of 26 triples on the 13 points of the set $A$ form a sub-STS(13) of the STS(27). As stated in the proof of Theorem 4.1, both STS(13)s have a set of three parallel blocks for which there does not exist a block intersecting all three.

(ii) $I(D) \leq 12$. The proof in this case is as in case (a). The number of $C_{14}$ configurations, $c_{14}>0$. Let $\{a b c, d e f, a d j, c e j\}$ be a $C_{14}$ configuration and define the configuration $\mathcal{C}$ precisely as in the proof of Theorem 4.2 . Then $p(\mathcal{C}) \leq 14$ and the set of the remaining 13 or more points is not an independent set and therefore contains a block ghi which is parallel to $a b c$, def, and such that criterion (iii) of Theorem 4.1 is violated.

(iii) $I(D)=13$ and the $\operatorname{STS}(27)$ is not 5-sparse. Then the $\operatorname{STS}(27)$ contains either a Pasch configuration or a mitre configuration.

In the former case, let the Pasch configuration be $\{a d m, a e n, b d n, b e m\}$. The set of pairs $\{a b, d e, m n\}$ yields a set of three blocks. If the third point of all of these blocks is the 
same, then the $\operatorname{STS}(27)$ contains a subsystem $\operatorname{STS}(7)$ and so cannot have a 3-existentially closed block intersection graph. Otherwise, possibly by relabelling the points, consider the pair of parallel blocks $a b c$, def, and define the configuration $\mathcal{C}$ precisely as in the proof of Theorem 4.2. Then $p(\mathcal{C}) \leq 13$.

In the latter case, let the mitre configuration be $\{a b c, d e f, a d j, b e j, c f j\}$. Again, define $\mathcal{C}$ precisely as in the proof of Theorem 4.2. Again, $p(\mathcal{C}) \leq 13$.

In both cases the set of the remaining 14 or more points is not an independent set and therefore contains a block $g h i$ which is parallel to both $a b c$, def, and such that criterion (iii) of Theorem 4.1 is violated.

(iv) $I(D)=13$ and the $\operatorname{STS}(27)$ is 5 -sparse. We show with the help of a computer that no such system exists.

Partition the points of the $\operatorname{STS}(27)$ into two sets $A$ and $B$ where $B$ is a maximum independent set. Then, using the same notation as in the proofs of Theorems 3.1 and 4.1, $\nu(1,2)=78, \nu(2,1)=13$ and $\nu(3,0)=26$. Now consider these $26 A A A$ triples on the $14 A$ points. Trivially, this is also 5-sparse. By an elementary counting argument, there exists a point of degree at most five. Remove this point and all the blocks containing it. What remains is a configuration containing 21 or more triples on 13 or fewer points. If the configuration contains more than 21 triples, then remove triples arbitrarily so that precisely 21 triples remain. Then, since a configuration on 12 or fewer points contains at most 20 triples, we have a configuration with precisely 21 triples on 13 points. Again, it is trivial that this is 5-sparse. We now repeat the above procedure of removing a point and all the blocks containing it; in this case a point of degree at most 4 . What remains is a configuration containing 17 or more triples on 12 or fewer points, again 5 -sparse.

At this stage the problem becomes computationally feasible. There are no 5-sparse configurations containing 18 or more triples on 12 or fewer points but there are four such configurations containing 17 triples on 12 or fewer points. We can then consider extensions of these four configurations. On 13 points, the numbers of non-isomorphic 5-sparse configurations containing 18, 19, 20 and 21 triples are respectively 39, 91, 34 and 0 . This completes the proof of the theorem.

In fact, using similar but more refined arguments to those employed in the above theorem to deal with the case $v=27$, we are able to settle the case for $v=25$. But since the proof is lengthy, and also requires a computer search, we omit the details here. They will appear in the first author's Ph.D. thesis [9]. But for completeness we state the result as a theorem.

Theorem 4.4 A Steiner triple system of order 25 cannot have a 3-existentially closed block intersection graph.

\section{$5 \quad$ The cases $v=19$ and $v=21$}

The results of the previous section imply that the block intersection graph of an STS $(v)$ can be 3-e.c. only if $v=19$ or 21. The number of non-isomorphic STS(19)s is known, [18]; 
there are 11,084,874,829 of them. We have systematically examined all 164,758 which have a non-trivial automorphism group and found that precisely two of them have 3-e.c. block intersection graphs. The first of these is a cyclic system, \#A3 in the listing of [17], given by the set of triples

$$
\begin{aligned}
&\{\{i, 1+i, 8+i\}: i=0,1, \ldots, 18\} \\
& \cup\{\{i, 2+i, 5+i\}: i=0,1, \ldots, 18\} \\
&\cup \quad\{\{i, 4+i, 10+i\}: i=0,1, \ldots, 18\}\},
\end{aligned}
$$

addition being performed modulo 19. The system has full automorphism group of order 57 and is not the well-known Netto system on 19 points. The second system has automorphism group of order 8 and consists of the set of 57 triples

$$
\begin{gathered}
\{012,034,135,236,146,245,057,068,569,178,279,37 a, 47 b, 67 c, 28 d, \\
38 e, 48 a, 58 f, 09 f, 19 g, 39 c, 49 h, 89 i, 0 a e, 1 a b, 2 a h, 5 a i, 6 a g, 9 a d, 0 b g, \\
2 b f, 3 b i, 5 b d, 6 b h, 8 b c, 9 b e, 0 c h, 1 c d, 2 c i, 4 c e, 5 c g, a c f, 0 d i, 3 d g, 4 d f, \\
6 d e, 7 d h, 1 e f, 2 e g, 5 e h, 7 e i, 3 f h, 6 f i, 7 f g, 4 g i, 8 g h, 1 h i\} .
\end{gathered}
$$

The block intersection graphs of non-isomorphic STS $(v)$ s are themselves non-isomorphic for $v \geq 19$ (see Corollary 2.3 of [18]). We have not systematically examined the STS(19)s with trivial automorphism group but we have generated millions of STS(19)s by the method of hill-climbing, [20], and analysed these. It would be expected that the vast majority would have trivial automorphism group but we found none which have a 3-e.c. block intersection graph. We also tried to construct STS(19)s with 3-e.c. block intersection graphs with the help of a computer, and in fact this is how we initially discovered the above system with automorphism group of order 8. But no others appeared. We have also examined all 2591 anti-Pasch STS(19)s and, again, none has a 3-e.c. block intersection graph.

The enumeration of non-isomorphic STS(19)s, [18], renders a complete analysis of the case $v=19$ feasible, although it would represent a very large computational task. No enumeration of non-isomorphic STS(21)s exists, nor is one likely to appear in the near future. Furthermore, the arguments used to dispose of the cases $v=27$ above and $v=25$ in [9], when applied to the case $v=21$, result in subcases for which the analysis by exhaustive techniques or by the techniques described in the present paper is currently infeasible.

None of the seven cyclic STS(21)s have 3-e.c. block intersection graphs. Also the computer methods which we applied to the STS(19)s failed to identify any STS(21) with a 3-e.c. block intersection graph. In fact we have not been able to find an STS(21) whose block intersection graph is in any way 'close' to being 3-e.c. 


\section{Acknowledgement}

The authors would like to thank Petteri Kaski and Patric Östergård, Helsinki University of Technology, for making available to us listings of Steiner triple systems of order 19.

\section{References}

[1] W. Ananchuen and L. Caccetta, On the adjacency properties of Paley graphs, Networks 23 (1993), 227-236.

[2] C. A. Baker, A. Bonato and J. M. N. Brown, Graphs with the 3-e.c. adjacency property constructed from affine planes, J. Combin. Math. Combin. Comput. 46 (2003), 65-83.

[3] A. Blass, G. Exoo and F Harary, Paley graphs satisfy all first-order adjacency axioms, J. Graph Theory 5 (1981), 435-439.

[4] A. Bonato, W. H. Holzmann and H. Kharagani, Hadamard matrices and strongly regular graphs with the 3-e.c. adjacency property, Electron. J. Combin. 8 (2001), 9pp.

[5] B. Bollobás and A. Thomason, Graphs which contain all small graphs, European J. Combin. 2 (1981), 13-15.

[6] L. Caccetta, P. Erdős and K. Vijayan, A property of random graphs, Ars Combin. 19 (1985), 287-294.

[7] C. J. Colbourn, E. Mendelsohn, A. Rosa and J. Šráŭ, Anti-mitre Steiner triple systems, Graphs and Combin. 10 (1994), 215-224.

[8] P. Erdős and A. Rényi, Asymmetric graphs, Acta. Math. Acad. Sci. Hungar. 14 (1963), 295-315.

[9] A. D. Forbes, Configurations and colouring problems in block designs, Ph.D. thesis, The Open University, UK (in preparation).

[10] A. D. Forbes, M. J. Grannell and T. S. Griggs, Configurations and trades in Steiner triple systems, Australas. J. Combin. 29 (2004), 75-84.

[11] Y. Fujiwara, Constructions for anti-mitre Steiner triple systems, J. Combin. Des. (to appear).

[12] Y. Fujiwara, Infinite classes of anti-mitre and 5-sparse Steiner triple systems, J. Combin. Des. (to appear).

[13] M. J. Grannell and T. S. Griggs, Configurations in Steiner triple systems, in: Combinatorial designs and their applications, ed. F. C. Holroyd, K. A. S. Quinn, C. Rowley and B. S. Webb, Chapman \& Hall CRC Research Notes in Math. 403 (1999), 103-126. 
[14] M. J. Grannell, T. S. Griggs and E. Mendelsohn, A small basis for four-line configurations in Steiner triple systems, J. Combin. Des. 3 (1995), 51-59.

[15] M. J. Grannell, T. S. Griggs and C. A. Whitehead, The resolution of the anti-Pasch conjecture, J. Combin. Des. 8 (2000), 300-309.

[16] A. C. H. Ling, C. J. Colbourn, M. J. Grannell and T. S. Griggs, Construction techniques for anti-Pasch Steiner triple systems, J. London Math. Soc. (2) 61 (2000), 641-657.

[17] R. A. Mathon, K. T. Phelps and A. Rosa, Small Steiner triple systems and their properties, Ars Combin. 15 (1983), 3-110.

[18] P. Kaski and P. R. J. Östergård, The Steiner triple systems of order 19, Math. Comp. 73 (2004), no. 248, 2075-2092 (electronic).

[19] N. Saur and J. Schönheim, Maximal subsets of a given set having no triple in common with a Steiner triple system on the set, Canad. Math. Bull. 12 (1969), 777-778.

[20] D. R. Stinson, Hill-climbing algorithms for the construction of combinatorial designs, Ann. Discrete Math. 26 (1985), 321-334.

[21] D. R. Stinson and Y. J. Wei, Some results on quadrilaterals in Steiner triple systems, Discrete Math. 105 (1992), 207-219.

[22] A. Wolfe, The resolution of the anti-mitre Steiner triple system conjecture, J. Combin. Des. (to appear). 


\section{Corrigendum - submitted November 27, 2006}

It has come to our attention that the statement of Theorem 4.1 is not quite correct. Criterion (iii) in that theorem should be amended to read as follows:

(iii) for every set of three parallel blocks, if $v<19$ there exists a block which is parallel to all three, and if $v \geq 19$ there exists a block which intersects all three.

There is no consequent change to the proof of the theorem nor to the remainder of the paper. All results remain valid. 